\title{
MultileVEl ANALYSIS OF STUDENT'S FEEDBACKUSING MOODLE LOGS IN VIRTUAL Cloud Environment
}

\author{
Ashok Verma ${ }^{1}$, Sumangla Rathore ${ }^{2}$, Santosh Vishwakarma ${ }^{3}$ and Shubham \\ Goswami $^{4}$ \\ ${ }^{1}$ Department of Computer Science \& Engineering, Sir Padampat Singhania University, \\ Udaipur, Rajasthan, India \\ ${ }^{2}$ Department of Computer Science \& Engineering, Sir Padampat Singhania University, \\ Udaipur, Rajasthan,India \\ ${ }^{3}$ Department of Computer Science \& Engineering, Gyan Ganga Institute of \\ Technology \& Sciences, Jabalpur, India \\ ${ }^{4}$ Department of Computer Science \& Engineering, Sir Padampat Singhania University, \\ Udaipur, Rajasthan, India
}

\begin{abstract}
In the current digital era, education system has witness tremendous growth in data storage and efficient retrieval. Many Institutes have very huge databases which may be of terabytes of knowledge and information. The complexity of the data is an important issue as educational data consists of structural as well as non-structural type which includes various text editors like node pad, word, PDF files, images, video, etc. The problem lies in proper storage and correct retrieval of this information. Different types of learning platform like Moodle have implemented to integrate the requirement of educators, administrators and learner. Although this type of platforms are indeed a great support of educators, still mining of the large data is required to uncover various interesting patterns and facts for decision making process for the benefits of the students.

In this research work, different data mining classification models are applied to analyse and predict students' feedback based on their Moodle usage data. The models described in this paper surely assist the educators, decision maker, mentors to early engage with the issues as address by students. In this research, real data from a semester has been experimented and evaluated. To achieve the better classification models, discretization and weight adjustment techniques have also been applied as part of the pre processing steps. Finally, we conclude that for efficient decision making with the student's feedback the classifier model must be appropriate in terms of accuracy and other important evaluation measures. Our experiments also shows that by using weight adjustment techniques like information gain and support vector machines improves the performance of classification models.
\end{abstract}

\section{KEYWORDS}

Educational Data, Educational Data Mining,LMS, Moodle, Feedback system, weight adjustment techniques. 


\section{INTRODUCTION}

The use of web-based education systems or e-learning systems has grown exponentially in the last years, spurred by the fact that neither students nor teachers are bound to any specific location and that this form of computer-based education is virtually independent of a specific hardware platform. In particular, collaborative and communication tools are also becoming widely used in educational contexts and as a result. Learning Management Systems (LMSs) are becoming much more common in universities, community colleges, schools, and businesses, and are even used by individual instructors in order to add web technology to their courses and supplement traditional face-to-face courses. LMSs can offer a great variety of channels and workspaces to facilitate information sharing and communication among participants in a course. They let educators distribute information to students, produce content material, prepare assignments and tests, engage in discussions, manage distance classes and enable collaborative learning with forums, chats, file storage areas, news services, etc. Some examples of commercial systems are Blackboard and TopClass while some examples of free systems are Ilias,Claroline and Moodle. Cloud computing can store a huge amount of educational resources and provide infrastructure, platform, and application services for users instead of letting users save them in their devices. It can also provide unlimited computing power for the completion of various types of application [1][2].

Nowadays, one of the most commonly used Learning Management System is Modular Object Oriented Dynamic Learning Environment (Moodle), a free learning management system enabling the creation of powerful, flexible and engaging online courses and experiences. The following figure shows an architecture of Moodle system which is based on a layered approach.

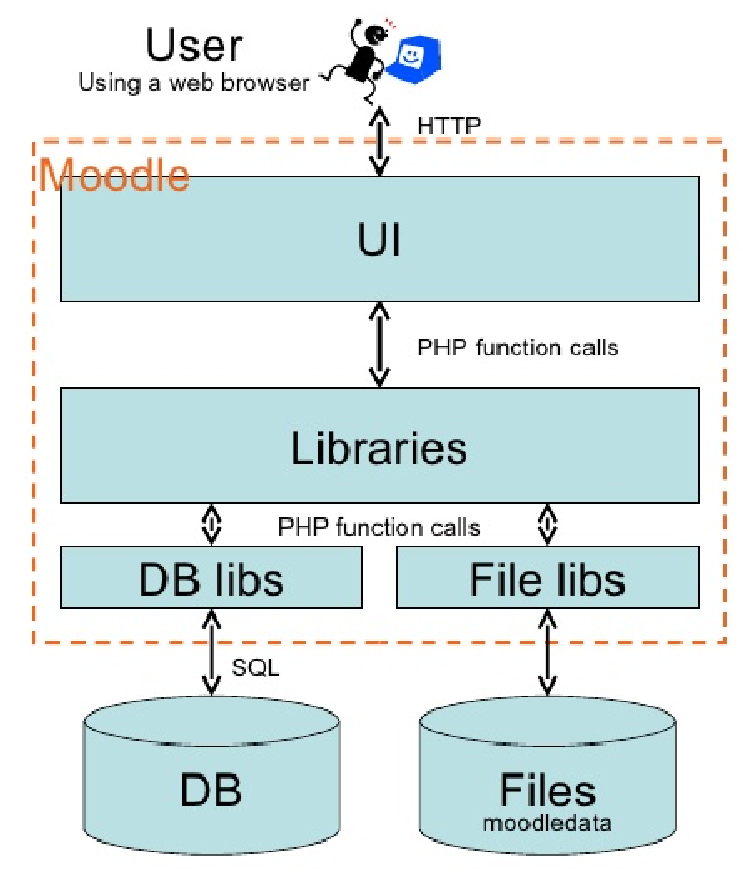

Figure 1.Architecture of Moodle System 
Moodle (Modular Object-Oriented Dynamic Learning Environment) is a mainstream open-source learning management system widely welcomed due to its simple and clear operation as well as flexible space expanding capability [24]. The typical working environment is a server with LAMP, short for Linux operation system, Apache server, Mysql database and PHP scripting language. This architecture could optimize Moodle and makes it more stable and safer. A Moodle Virtual Cluster is constructed through virtualization technology in cloud computing to spread excessive load in single server of Moodle and avoid aforesaid shortcomings in physical cluster. Virtual cluster can dynamically allocate resources on demands, thus utilizing resources efficiently and constructing an elastic computing architecture [5][17].

Moodle has benefited many Universities and colleges around the world. Moodle allows teachers to assess hundreds and thousands of students' performance as it allows the instructors to track students' grades, personal information, academic results, number of tests taken, and other activities. This data is usually termed as Educational data. The data collected from Students' is huge and manually extracting any useful information from such huge dataset is not an easy task. One such approach which can be used here is Data Mining. Data Mining is a technique of extracting useful and hidden information from large databases. It is also termed as a "Knowledge discovery" process. This knowledge once mined can be used to increase revenues, sales, cut costs or both. [19].

In the past few years, researchers have largely focused on using Data Mining techniques such as classification, clustering, association rule mining to analyse educational data; i.e. to improve the educational system. These methods have been proven immensely useful in analysing students' learning behaviour and performance. Data mining techniques have been successfully applied to educational data; to understand students' learning issues, recommendation system: where new courses are recommended on the basis of their performance, feedback for teachers, etc. Though there are several tools which can be used for analysis; but they have not given much insight into students' behaviour. The educational data can be mined to understand students' behaviour using data mining techniques; this is termed as Educational Data Mining.

\section{RELATED WORK}

The problem of high computational load in Moodle server has been addressed by Guo et al. [1]. They proposed that the centralized server load must be allocated to several other servers in terms of virtual clusters. They perform concurrent access pressure test to evaluate the virtual cloud clusters performance and found that it improves transaction capability of the system.

Chen et al. [4] proposed an efficient resource management system for on-line virtual clusters provision, aiming to provide immediately-available virtual clusters for academic users. Particularly, they investigated two crucial problems: efficient VM image management and intelligent resource mapping, either of them has remarkable impact on the performance of the system.

Another important work carried out by [20] about the usage of data mining applied for personalization in web environments. They also develop tools for web based learning environment primarily focused on evaluating learning process of the educational system.

A similar work is carried out by [21] which focus on web-based technology that relates affordability of accessing the ubiquitous Web and the simplicity of deploying and maintaining 
resources. They discussed some data mining and machine learning techniques that could be used to enhance web-based learning environments for the educator to better evaluate the leaning process, as well as for the learners to help them in their learning endeavour.

Another approach as suggested by Alves et al. [16] is to extends a recent comparative study covering four different courses lectured at the Polytechnic of Porto - School of Engineering, in respect to the usage of a particular Learning Management System, i.e .Moodle, and its impact on students' results.

A similar approach was proposed by Daraghmi et al. [23]. They suggested that a new method for Moodle a web based interface supporting a wide range of activities including forums, contents, and assignments is provided to assist both the teachers and the students. However, limitations of the file-size acceptable for uploads, weakness in the assessment procedure, complicated task of replacing an existing file, and the lowest visiting rate of the traditional discussion module are some major deficiencies in the traditional Moodle module. They also integrated Moodle with Facebook to increase the visiting rate of the traditional discussion Moodle module, hence, increasing the students' motivation to ask questions and the interaction among the students. The performance and the usability of the new module were evaluated and promising results were obtained.

Another seminal work by Nagi\&Suesawaluk [18], suggested that virtual learning environment is designed to help educators create online courseware with opportunities for rich interaction. Interactivity is becoming a key facet of eLearning. Moodle logs all activities including views and posts for all learning objects hosted in the system. It provides different statistics to help the content experts to improve the quality of eLearning courseware. They also describes the use of automated, scalable real-time containing data of all activity for four major ICT courses offered as a part of the Master Degree eLearning program at Assumption University of Thailand.

Recently Holbl et al. [19] presented experience with learning management system Moodle when used in their educational process. They discussed the use of a feedback form enabling students to asses and comment courses. Additionally, the results of a questionnaire compiled to gain data on student experiences with Moodle with focus on features of the platform and specific privacy concerns are presented. Further, the relation between the experience gained with the course and the questionnaire results is described. Students were asked about Moodle features they use and specific privacy concerns, including visibility of profiles, results and grades. Also students' relation to giving feedback feature of Moodle user's analysed.

Another important work on web usage mining with multilevel analysis and data pre-processing is given by Sael et al. [20]. This research illustrates the potential of Web Usage Mining on eLearning domain. They used educational data mining techniques to analyse learners' behaviour, to help in learning evaluation and to enhance the structure of a given course. They focused on the pre-processing task, which is considered as the most crucial phase in the whole process. They also presented multidimensional graphics in order to understand users' accesses. These aggregated variables provide teachers and tutors with interesting knowledge about students' learning process according to different levels of content accessed.

Recently an important work is carried out by Pong \&Rungworawut [21] which focus on the new pattern of teaching evaluation. The research carried out by them also presents analysis model for 
teaching evaluation from answering and posting a comment to discussion in form of open-end question obtained from Moodle LMS forum using data mining techniques. The techniques extract classification of attitudes that are defined positive and negative attitude from students to instructor for improvement of learning and teaching.

Another important paper is given by Gil et al. [22]. They proposed a new way to verify the identity of users in learning management systems used at Higher Education. The starting point is to study the needs demanded by the new Distance Education model. After the study of possible security risks in the new environment, they conclude that the use of biometrics tools provide a good mechanism to check the user identity. In this article the authors show how to integrate a fingerprint verification system in an open source LMS called Moodle as a reliable method for achieving user authentication. The system was used successfully for a group of Industrial Engineering students at UNED for accessing their Electronic laboratory exams. They also analysed the students' attitudes faced with a new way of control access both from the technical and personal points of view.

Based on the extensive literature survey carries out, our proposed work focus on first; the preprocessing step with the Moodle corpus as an important and key aspects before analysing the students feedback pattern and second; the recommendation based as the solution of the problems identified.

\section{Methodology}

For assessment of our approach, we utilized the information from the Moodle framework. Moodle, like most LMSs, records every one of the understudies' utilization data in log files as well as straightforwardly in a database. There exists large number of interrelated relations in the Moodle database. Be that as it may, this data is not required thus it is additionally important to change over the valuable information to the required arrangement utilized for the mining purpose. Therefore, Moodle information must be pre-processed to change over it to the adoptable format for further processing and mining. Then, various data mining algorithms (classification algorithms in our case) will be executed to find hidden patterns and data inside the info of interest for the instructor. So, this mining method consists of three phases of knowledge discovery process: pre-processing, data processing, and post-processing. Next there is an overview in further detail relating to how these steps have been perform with the Rapidminer tool used in this research.

In the pre-processing step, the user created data files from the Moodle database have been imported in excel worksheet.Our mining tool additionally splits the data file into parts, such as training and testing files. Table 1 shows the list of attributes used for processing of the Moodle data. The feedback of students have been assessed with course name as identification of course, N_post as the total number of posts submitted by student in a current semester, N_post sub is the attribute which signify the number of posts related to teaching feedback and N_post teacher specify the post which relates teachers feedback. 
International Journal of Computer Science \& Information Technology (IJCSIT) Vol 10, No 5, October 2017

Table 1. Attributes used by students in Moodle Logs

\begin{tabular}{|l|l|}
\hline \multicolumn{2}{|l|}{ Attributes used for students } \\
\hline Name & Description \\
\hline Course & Identification number of the course \\
\hline N_post & No of posts send to Moodle \\
\hline N_post_sub & No of subject related posts send to Moodle \\
\hline N_post_teacer & No of teacher related posts send to Moodle \\
\hline
\end{tabular}

As specify in the Figure 2, Moodle database has been integrated and several preprocessing steps have been performed as part of preprocessing. The major steps includes tokenization, stop word removal, stemming and generate n-grams during pre-processing of data. Tokenization [3] refers to process of taking character sequence from defined document unit and breaking it into words, symbols, phrases and numbers called tokens. Stop word removal [4] filter out the words that have no values for retrieval purpose. Stemming [5] perform replacement of all the variations of the words with its root word. The variant words may be plurals, gerund forms, prefixes, suffixes etc. a stem word can represents all of its variants that reduces the size of dictionary containing all words of document collection. In our analysis Porter algorithm found best because it produces maximum number of tokens.

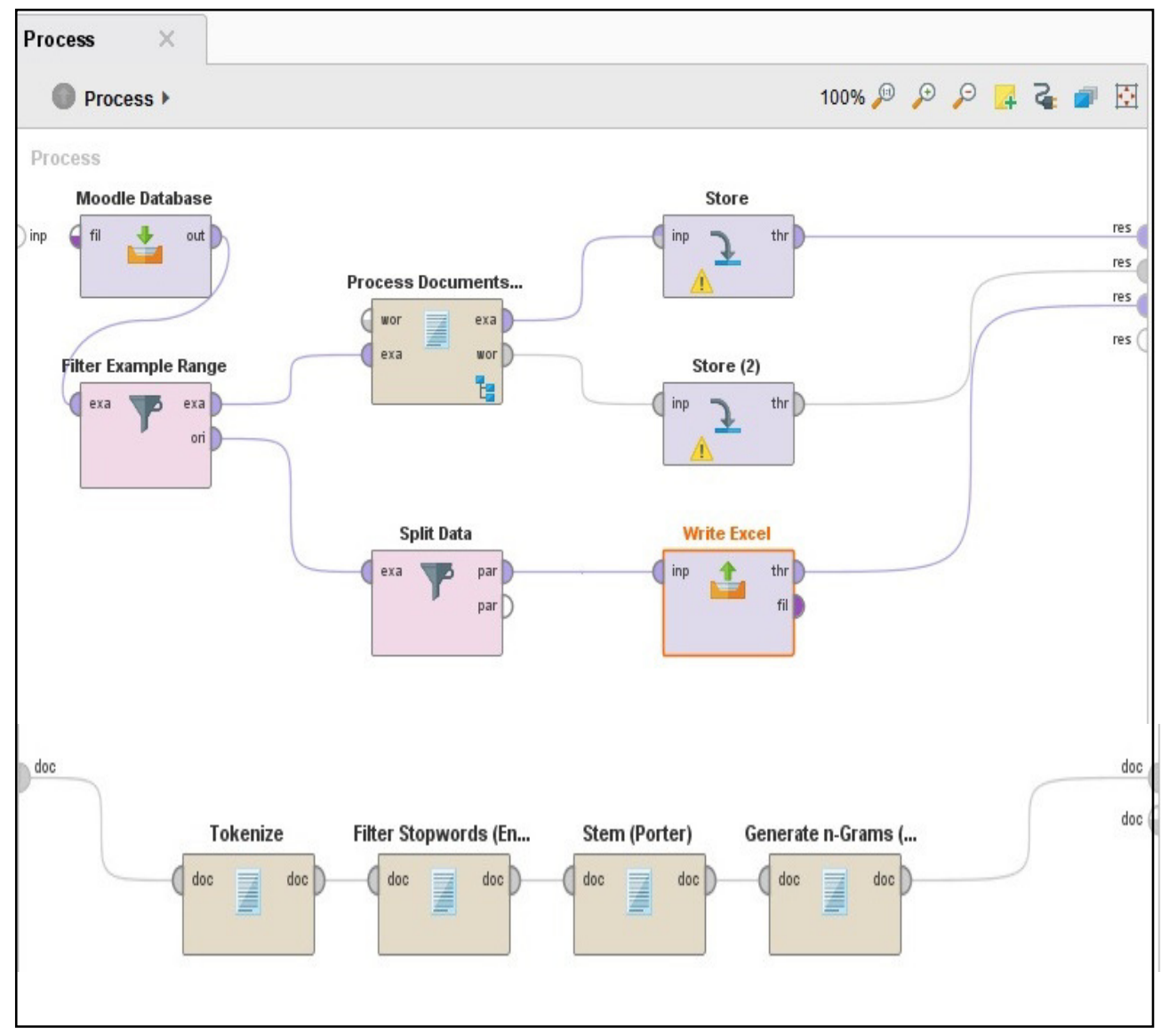

Figure 2 Import of Moodle database and preprocessing 
The output from the process as shown in Figure 2 generates the document vector and a word list. We store the word list and document vector generated by above step for future use. The document vector is further process through weight adjustment as shown in Figure 3 below,

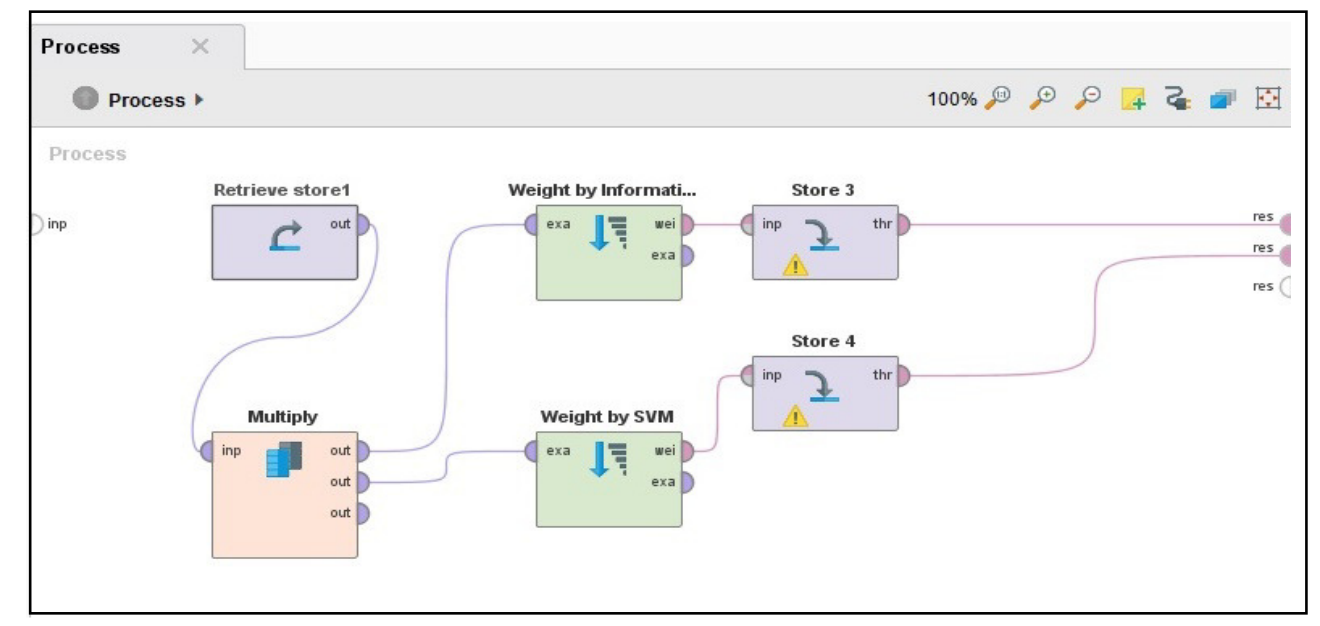

Figure3 Term weight adjustment by Information Gain and SVM

We deploy two weight adjustment methods for our document vector which strive the purpose of ranking the terms according to their discriminating power and normalize the term weight with compare to the reference values. First the weight by Information Gain method uses the information gain ratio of label attribute to calculate the value of un-label attribute and assigns the weight accordingly. Second, we used SVM method for weight adjustment which computes attributes weights with the coefficients of the normal vector of a linear SVM. The weight modification procedure brings about a sensible decrease in the modelling costs. This process generates two weighting tables as shown in Figure 3.

Once we have the updated document vector and attribute weights, we evaluated four state of the art classification models to understand which give the best accuracy as shown in Figure 4. The algorithms used are K-NN, Decision Tree, Random Forest,Generalized Linear Modeland Naive Bayes. The descriptions of the models are as following:

Random forest is an ensemble learning method for classification, in which it constructs multiple of unpruned classification trees in the training phase, by bootstrap sampling method on the training data. The final predicted output for a random selected feature is given by finding the mean from all unpruned classification trees in the testing phase [18].

Naive Bayes classification is the extended form of Bayesian classifiers which include naïve assumption too. Bayesian classifiers are statistical classifiers, which is based on Bayes' theorem. Bayesian classifiers can predict probability that a given sample belongs to a particular class, i.e. can predict class membership probabilities. According to the naïve assumption, the changes in an attribute value on a given class are independent of the changes in the values of the other attributes. This assumption is also known as 'class conditional independence' [19]. 
Suppose there are $\mathrm{k}$ different classes denoted as $\mathrm{C} 1, \mathrm{C} 2 \ldots \mathrm{Ck}$. Let $\mathrm{X}=\{\mathrm{x} 1, \mathrm{x} 2 \ldots \mathrm{xn}\}$, depicting $\mathrm{n}$ measured values of the $\mathrm{n}$ attributes, $\mathrm{A} 1, \mathrm{~A} 2 \ldots$ An respectively. Then $\mathrm{X}$ is predicted to belong to the class $\mathrm{Ci}$ if and only if

$$
P(C i \mid X)>P(C j \mid X) \quad \text { for } 1 \leq j \leq m, j \neq i .
$$

Where $\mathrm{P}(\mathrm{ClX})$ is the posterior probability of class $\mathrm{C}$ conditioned on predictor $\mathrm{X}, \mathrm{P}(\mathrm{C})$ is the prior probability of class $\mathrm{C}, \mathrm{P}(\mathrm{X} \mid \mathrm{C})$ is the probability of predictor $\mathrm{X}$ conditioned on class $\mathrm{C}$ and $\mathrm{P}(\mathrm{X})$ is the prior probability of predictor X. By Bayes' theorem:

$$
\mathrm{P}(\mathrm{Ci} \mid \mathrm{X})=(\mathrm{P}(\mathrm{X} \mid \mathrm{Ci}) \mathrm{P}(\mathrm{Ci})) / \mathrm{P}(\mathrm{X})
$$

Rule induction uses sequential covering algorithm to extract the "if, then" rule, and they are directly extracted from the training data set. These rules are learned sequentially. One rule covers multiple examples present in the database hence, termed as the sequential covering algorithm. The collection of rules extracted represents full model. [4]

Decision trees are classifying model which can be directly transformed into a set of IF-THEN rules that are one of the most popular forms of knowledge representation, due to their simplicity and comprehensibility.

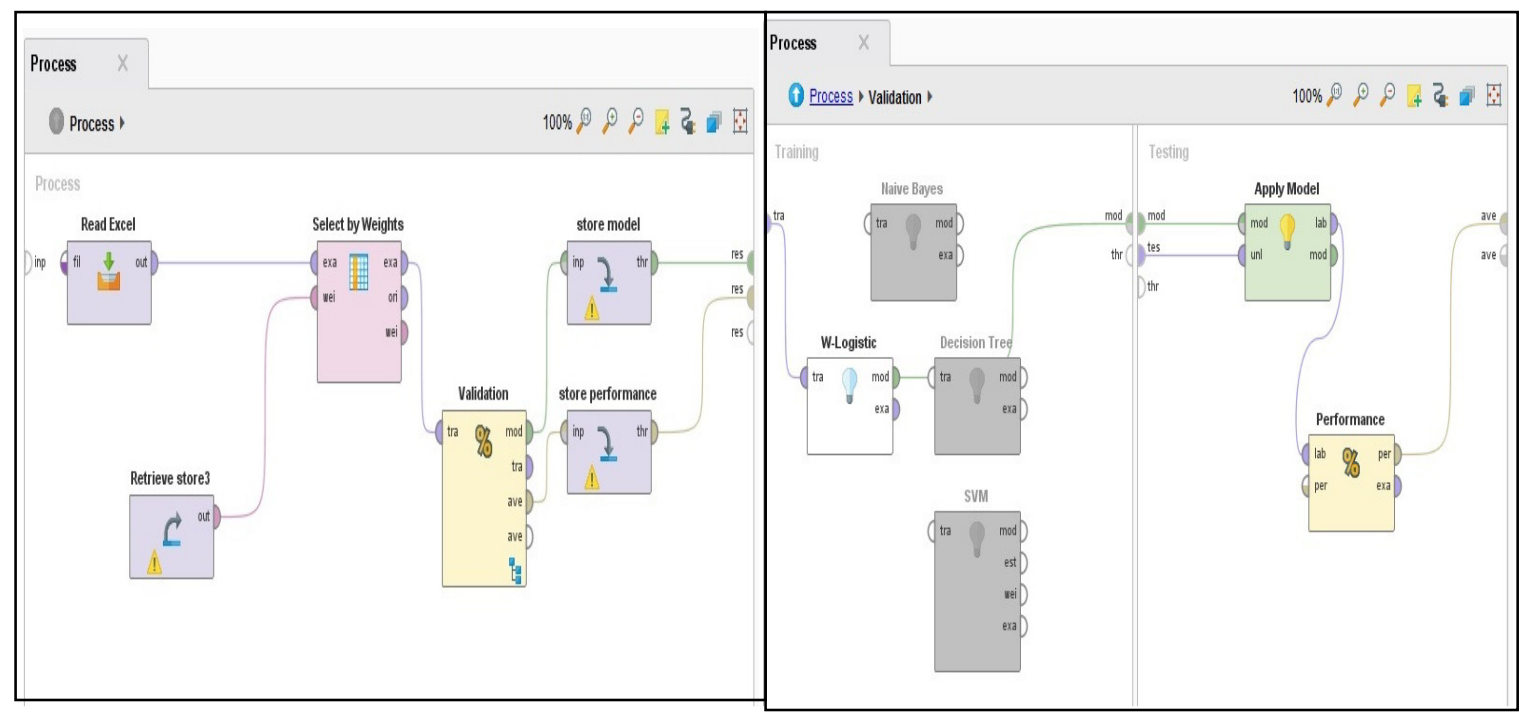

Figure 4: Various Classification Models

Generalized linear model perform predictions for a variable $\mathrm{Y}$ using a predictor variable $\mathrm{X}$; this is termed as simple linear regression. We model the relationship between the two independent and dependent variables. Generalized linear model is an extension of Linear Regression which allows variables with error distribution models other than allowing only variables with Normal Distribution as in the case of Linear Regression. In other words, we can say that GL Model is the generalized version of Linear Regression. GL model performs classification by making predictions for an unknown object for its target class. Mathematically, GL model is expressed as: 


$$
E(Y)=g^{-1}(X \beta)
$$

The parameters, $\mathrm{E}(\mathrm{Y})$ denotes the expected value/ target class for $\mathrm{Y}, \mathrm{X} \beta$ represents the predictor in linear form, $\mathrm{g}$ is the link function used by GL model for prediction.

\section{ReSUlt AND ANAlysis}

The Moodle database used in our experimentation consists of around 1500 records of University students. This record mimics various feedbacks related to various subjects and teachers. In this paper, the open source data mining tool Rapid Miner is used for experimentation. The split ration of 70:30 is used for training and testing of model performance. The models used in our experimentation have correctly classified their problems according to the feedback entered by them for various issues. It is very useful to analyse what issues students' face in their subjects and how it can be resolved. These predictions are automatically made by the system and no manual effort is required. The analysis shows that there are some common subjects' problems which students' face in their college curriculum. These issues must be resolved for their better academic career and future. Different classification models such as Naive Bayes, K-NN, Decision Tree, Random Forest, Generalized linear model are applied in the Moodle dataset after pre-processing and a comparative study has been made on the basis of their performance and accuracy in making predictions.

The table below shows the comparative study for various classification algorithms along with their performance parameters such as accuracy, classification error rate, Kappa, precision and recall.

Table2. Performance Table

\begin{tabular}{|c|c|c|c|c|c|}
\hline Classifier & $\begin{array}{c}\text { Naive } \\
\text { Bayes }\end{array}$ & K-NN & $\begin{array}{c}\text { Decision } \\
\text { Tree }\end{array}$ & $\begin{array}{c}\text { Random } \\
\text { Forest }\end{array}$ & $\begin{array}{c}\text { Generalized Linear } \\
\text { Model }\end{array}$ \\
\hline Accuracy & $96.00 \%$ & $98.50 \%$ & $98.12 \%$ & $93.87 \%$ & $99.00 \%$ \\
\hline Classification error & $4.00 \%$ & $1.50 \%$ & $1.88 \%$ & $6.13 \%$ & $1.00 \%$ \\
\hline Kappa & 0.935 & 0.975 & 0.969 & 0.894 & 0.983 \\
\hline Weighted mean recall & $97.24 \%$ & $98.80 \%$ & $98.38 \%$ & $91.87 \%$ & $98.89 \%$ \\
\hline $\begin{array}{c}\text { Weighted mean } \\
\text { precision }\end{array}$ & $96.29 \%$ & $98.60 \%$ & $98.36 \%$ & $96.76 \%$ & $99.38 \%$ \\
\hline
\end{tabular}

We can see from the above table that GL Model has given best performance in terms of achieving high accuracy and low classification error rate. K-NN, Decision Tree and Naive Bayes have also achieved high accuracy rates. Random forest classifier uses combination of decision trees with a random function for classifying data has given a little high error rate as it requires more features for data classification. This may not be appropriate in classifying students' subjects' problems from the Moodle dataset as we emphasize on the problems rather than different features. Classifiers which can effectively handle polynomial data have achieved high accuracy and better results. 
The graphs below shows the analysis for different classifiers using performance parameters -

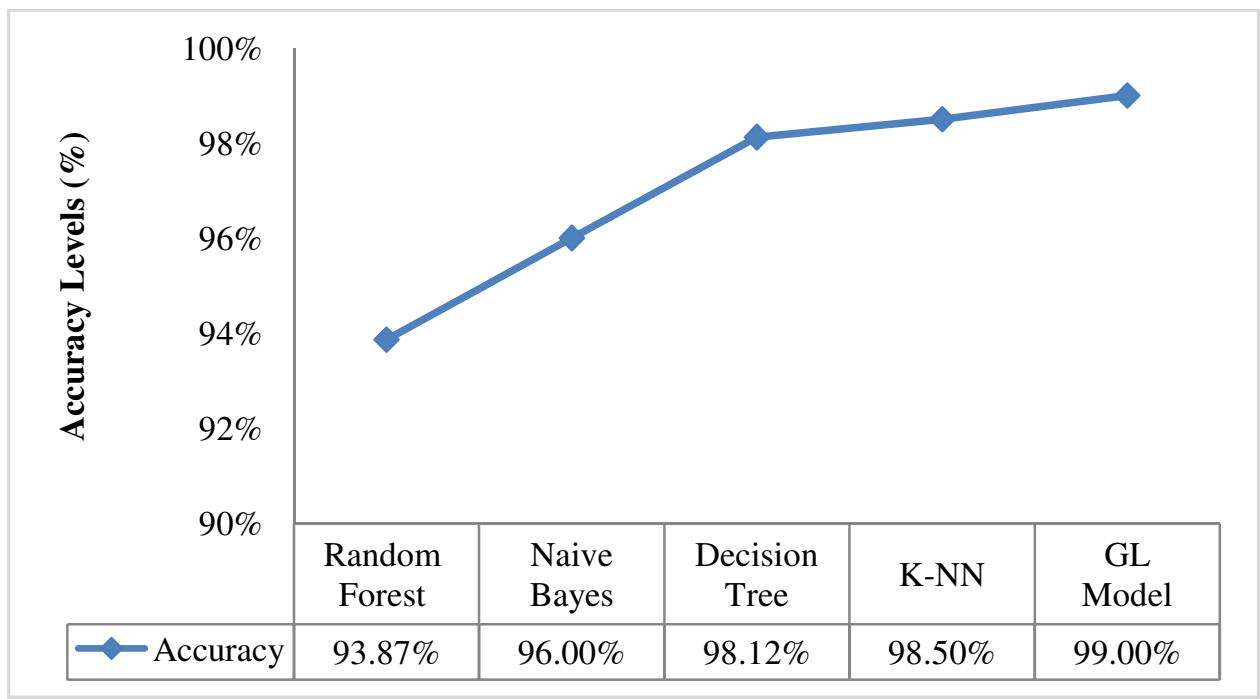

Figure 5. Performance measure of classifier using Accuracy

Accuracy is defined as how correct the predictions are made by the system. We can see from the above graph that GL Model has achieved highest accuracy of $99.90 \%$. Other classifiers have also achieved high accuracy results.

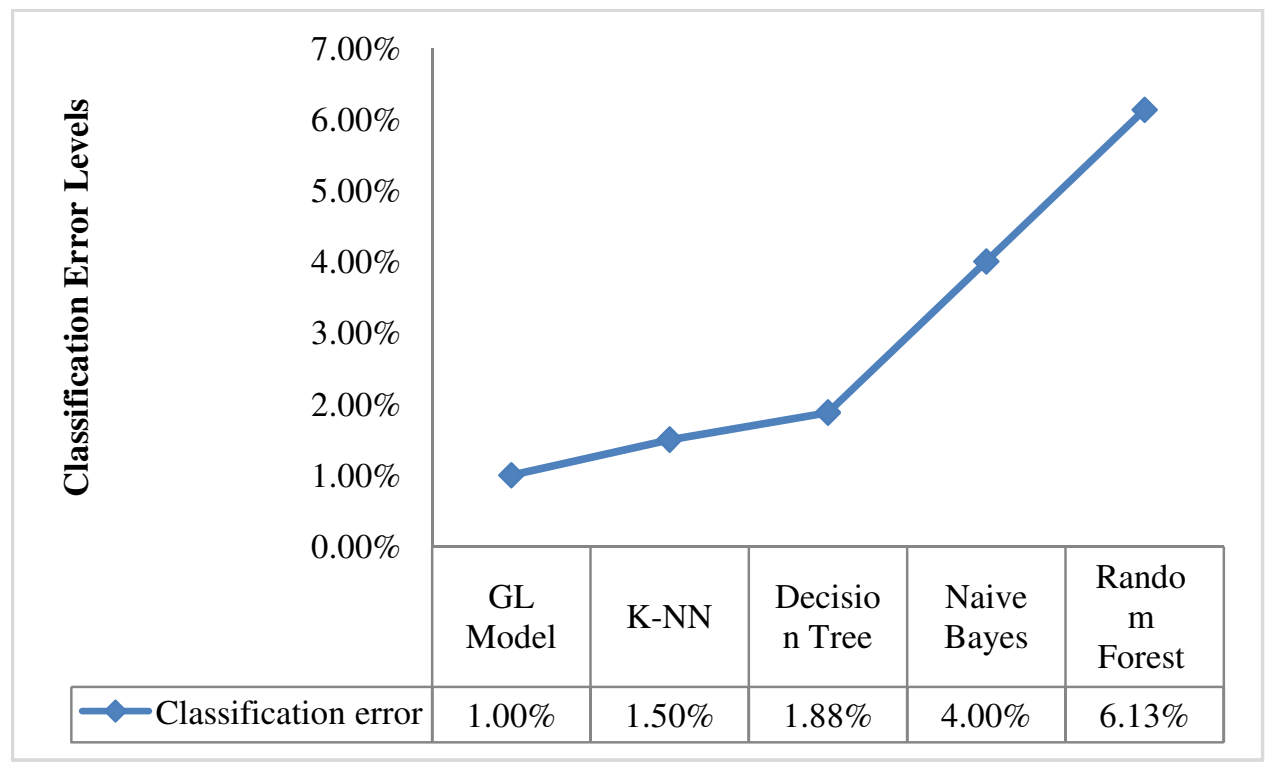

Figure 6 Performance measure of classifier using Classification Error

Classification error rate is the percentage of incorrect predictions made by the system. The graph above shows that GL Model has given least classification error rate of $1 \%$, as it achieved high accuracy rate. Other classifiers have also given less error rate; where only random forest has given little high error as it achieved low accuracy. 
International Journal of Computer Science \& Information Technology (IJCSIT) Vol 10, No 5, October 2017

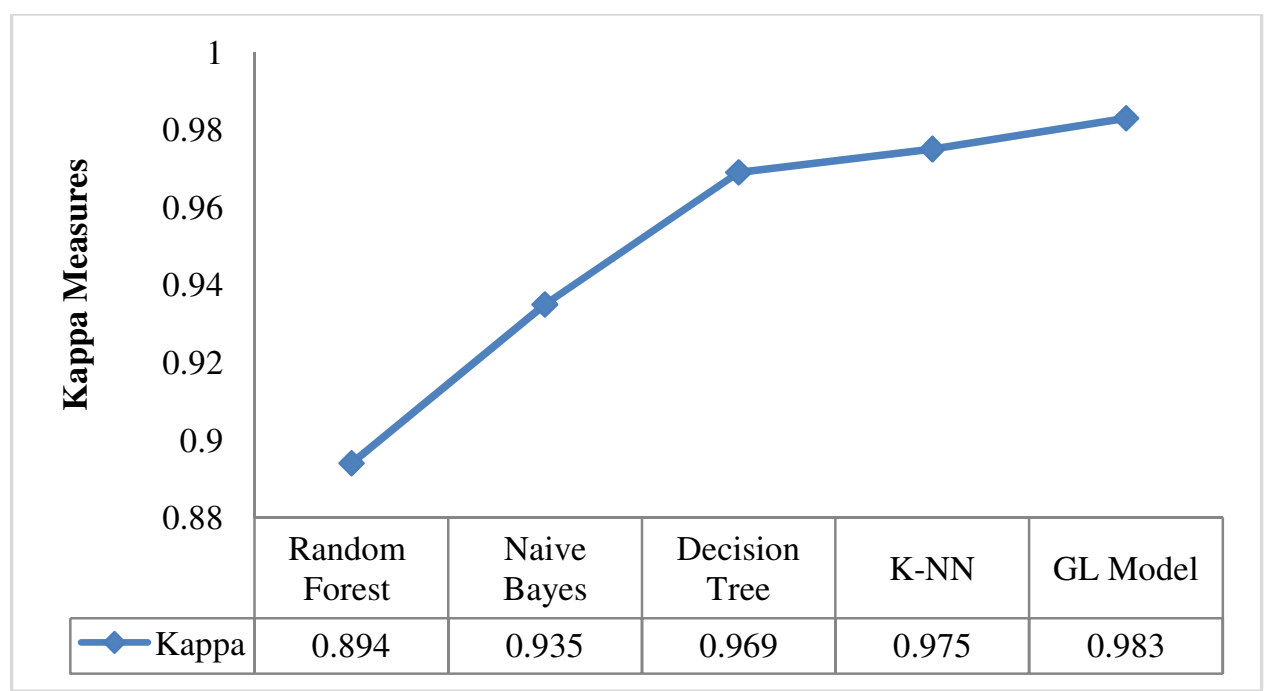

Figure 7. Performance measure of classifier using Kappa

Kappa statistics is a popular measure to check how correct the predictions are made by the system. It is considered more robust as compared to accuracy; as it also considers correct predictions made by 'chance' by the system. It compares the accuracy of predictions by the system with a random system. The analysis shows that GL Model has gained highest Kappa value of 0.983 which is close to 1 . Other classifiers have also achieved close to 1; high Kappa values.

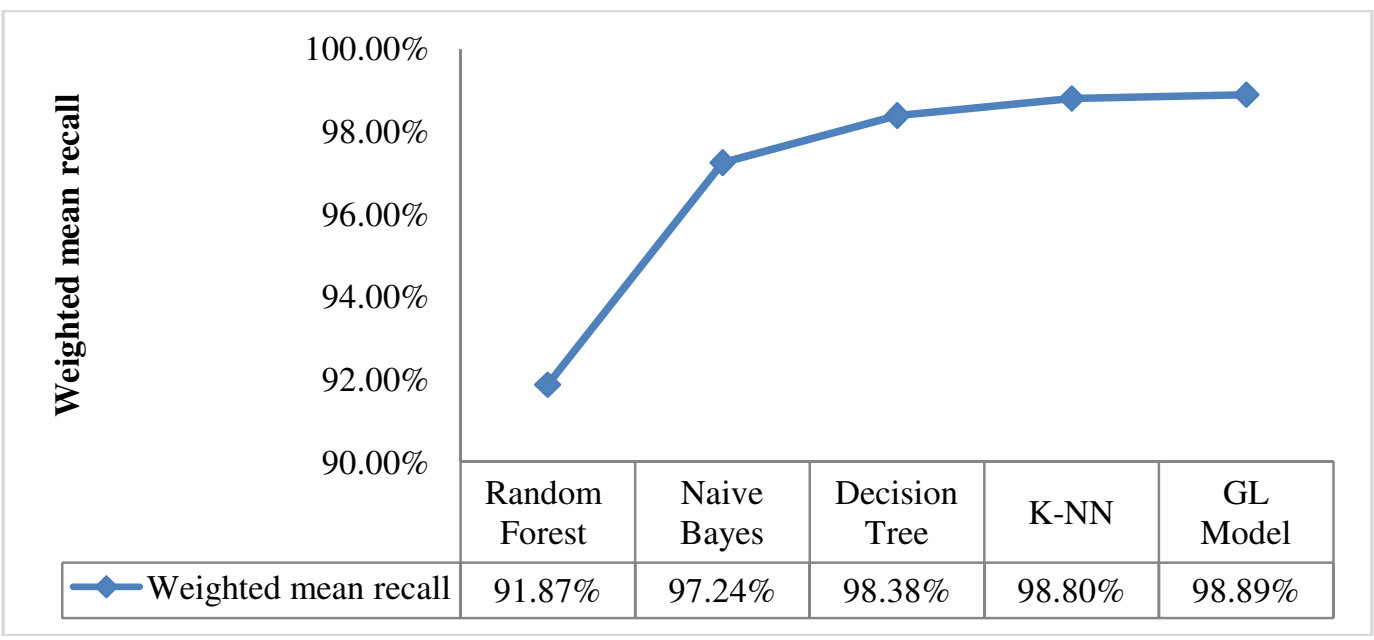

Figure 8. Performance measure of classifier using Kappa 


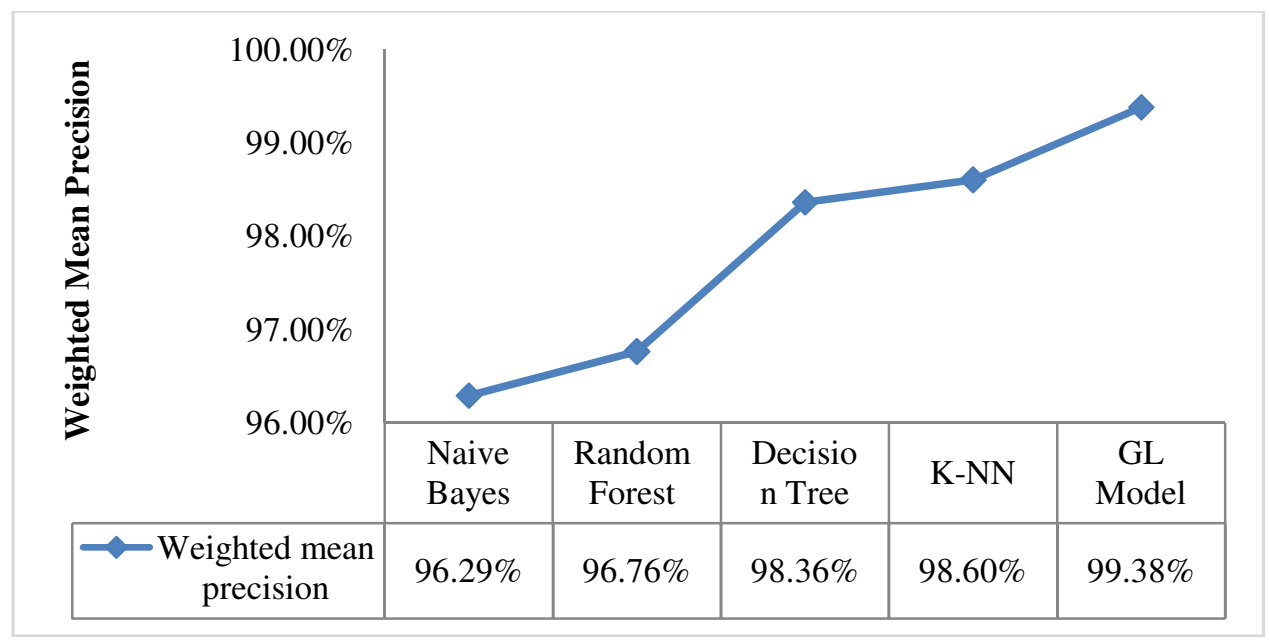

Figure 9 Performance measure of classifier using Weighted Mean Precision

Recall and Precision are also important measures to analyse the performance of data mining models. The weighted mean recall and precision for each individual class is calculated. The above analysis shows that GL Model has achieved high mean value of $98 \%$.

\section{CONCLUSION}

In this paper we had analysed student's feedback Moodle data with different data mining classification models with term weight adjustment schemes. It has been found that some algorithms shows better performance after applying various pre-processing tasks such as tokenization, stop word removal, stemming, etc. Further, by using weight adjustment by support vector machine and information gain also improves in terms of accuracy and other important evaluation measures. Although we have applied the techniques individually, but these models can also be applied in multi model scenario to obtain interesting patterns and knowledge in more accurate and faster way. The results obtain in our experiments are very useful to classify students problems as well as they are useful to detect other interesting patterns about the source of Moodle data. The main objective of our work was to improve the quality of student learning and to timely provide feasible solutions for their different problems.

In future experiments, we intend to measures on other important issues related to student profile, curriculum choices etc. The classification model used in our work will also be evaluated in terms of compressibility. By this we will be able to specify about the quantity and quality of the data can affect the performance of the algorithms. This paper can be further extended to increase the accuracy percentage by using several other classifiers at different numbers of folds. Finally, in order to prove the acceptability of the model accuracy, we look forwardto deploy the model with much larger dataset in real scenarios. 
International Journal of Computer Science \& Information Technology (IJCSIT) Vol 10, No 5, October 2017

\section{REFERENCES}

[1] XinGuo, Qing Shi, Danjue Zhang, “A Study on Moodle Virtual Cluster in Cloud Computing”, Internet Computing for Engineering and Science (ICICSE), 2013 Seventh International Conference , Publisher: IEEE

[2] M. Shiraz, S. Abolfazli, Z. Sanaei, and A. Gani, "A study on virtual machine deployment for application outsourcing in mobile cloud computing," The Journal of Supercomputing, vol. 63, March 2013, pp. 946-964, doi:10.1007/s11227-012-0846-y.

[3] Shiraz M, GaniA (2012) Mobile cloud computing: critical analysis of application deployment in Virtual machines. In: ICICN 2012 IPCSIT, 25-28 February, 2012. IACSIT Press, Singapore

[4] Chen, Yang, TianyuWo, and Jianxin Li. "An efficient resource management system for on-line virtual cluster provision." Cloud Computing, 2009.CLOUD'09.IEEE International Conference on.IEEE, 2009.

[5] Ye, Kejiang, et al. "Analyzing and modeling the performance in xen-based virtual cluster environment." High Performance Computing and Communications (HPCC), 2010 12th IEEE International Conference on.IEEE, 2010.

[8] J. Mostow and J. Beck, Some useful tactics to modify, map and mine data from intelligent tutors, Nat Lang Eng 12 (2006), 195-208, 16

[9] J. Mostow, J. Beck, H. Cen, A. Cuneo, E. Gouvea, and C. Heiner, An educational data mining tool to browse tutor-student interactions: Time will tell, In: Proceedings of theWorkshop on Educational Data Mining, 2005, pp 15-22.

[10] L.Dringus and T. Ellis, Using data mining as a strategy for assessingasynchronous discussion forums, Computer \& Education 45 (2005), 141-160. Elsevier, Science Direct.

[11] M.E. Zorrilla, E. Menasalvas, D. Marin, E. Mora, and J. Segovia, Web usage mining project for improving web-based learning sites, In Web Mining Workshop (2005), 1-22.

[12] O. Za“iane and J. Luo, Web usage mining for a better web-based learning environment, In: Proceedings of the Conference on Advanced Technology for Education, 2001, pp 60-64.

[13] C. Romero and S. Ventura, Educational data mining: A survey from 1995 to 2005, Expert SystAppl 33 (2007), 135-146.

[14] C. Romero and S. Ventura, Educational data mining: A review of the state-of- the-art, IEEE Trans Syst Man Cybern C (in press).

[15] OdedMaimon - LiorRokach, Data Mining and Knowledge Discovery Handbook Second Edition Springer 2010

[16] Alves G.R., Viegas M.C., Marques M.A., Silva, A.A., Costa-Lobo .C.,Formanski F., Silva, J.B. "Student performance analysis under different Moodle course designs", Interactive Collaborative Learning (ICL), 2012 15th International Conference on DOI: 10.1109/ ICL.2012.6402181 Publication Year: 2012, Page(s): 1 - 5

[17] Daraghmi, E.Y. ; Cheng Hsun Hsiao ; Shyan Ming Yuan "A New Cloud Storage Support and Facebook Enabled Moodle Module" Ubi-Media Computing and Workshops (UMEDIA), 2014 7th International Conference DOI: 10.1109/U-MEDIA.2014.12 Publication Year: 2014 , Page(s): 78 - 83 , 17

[18] Nagi, K. ;Suesawaluk, P. , "Research analysis of moodle reports to gauge the level of interactivity in elearning courses at Assumption University, Thailand"Computer and Communication Engineering, 2008. ICCCE 2008. International Conference DOI: 10.1109/ICCCE.2008.4580710 Publication Year: 2008, Page(s): 772 - 776,

[19] Holbl, M. ;Welzer, T. ; Nemec, L. ; Sevcnikar, A. "Student feedback experience and opinion using Moodle" Publication Year: 2011, Page(s): $1-4$

[20] Sael, N. ;Marzak, A. ; Behja, H. Web Usage Mining data preprocessing and multi level analysis on Moodle Computer Systems and Applications (AICCSA), 2013 ACS International Conference Publication Year: 2013 , Page(s): $1-7$

[21] Pong-Inwong, C. ; Rungworawut, W. Teaching evaluation using data mining on moodle LMS forum Information Science and Service Science and Data Mining (ISSDM), 2012 6th International Conference on New Trends Publication Year: 2012 , Page(s): 550 - 555 
International Journal of Computer Science \& Information Technology (IJCSIT) Vol 10, No 5, October 2017

[22] Gil, R. ;Sancristobal, E. ; Diaz, G. ; Castro, M. Biometric verification system in moodle\& their analysis in lab exams. International Conference on Computer as a tool. Publication Year: 2011, Page(s): $1-4$

[23] Gök, Abdullah, Alec Waterworth, and Philip Shapira. "Use of web mining in studying innovation."Scientometrics 102.1 (2015): 653-671.

[24] https://docs.moodle.org/33/en/About_Moodle

[25] www.rapidminer.com

[26] Altujjar, Yasmeen, et al. "Predicting Critical Courses Affecting Students Performance: A Case Study." Procedia Computer Science 82 (2016): 65-71

[27] Badr, Ghada, et al. "Predicting Students' Performance in University Courses: A Case Study and Tool in KSU Mathematics Department." Procedia Computer Science 82 (2016): 80-89.

[28] Barba, PG de, Gregor E. Kennedy, and M. D. Ainley. "The role of students' motivation and participation in predicting performance in a MOOC." Journal of Computer Assisted Learning 32.3 (2016): 218-231.

[29] Pursel, Barton K., et al. "Understanding MOOC students: motivations and behaviours indicative of MOOC completion." Journal of Computer Assisted Learning 32.3 (2016): 202-217. 